Voix et Images

\title{
Du plafond de verre et de la Révolution tranquille
}

\section{Lori Saint-Martin}

Volume 19, numéro 1 (55), automne 1993

Lionel Groulx écrivain

URI : https://id.erudit.org/iderudit/201079ar

DOI : https://doi.org/10.7202/201079ar

Aller au sommaire du numéro

Éditeur(s)

Université du Québec à Montréal

ISSN

0318-9201 (imprimé)

1705-933X (numérique)

Découvrir la revue

Citer cet article

Saint-Martin, L. (1993). Du plafond de verre et de la Révolution tranquille. Voix et Images, 19(1), 197-200. https://doi.org/10.7202/201079ar d'utilisation que vous pouvez consulter en ligne.

https://apropos.erudit.org/fr/usagers/politique-dutilisation/ 


\section{Du plafond de verre et de la Révolution tranquille}

\section{Lori Saint-Martin, Université du Québec à Montréal}

Le mot est lâché: c'est de pouvoir qu'il sera question. Ce fameux pouvoir politique, économique, social, qui échappe encore et toujours aux femmes. Deux publications très différentes - un essai sur l'histoire des femmes, un recueil de données statistiques sur la place qu'elles occupent dans les lieux de pouvoir ${ }^{1}$ - aboutissent à la même conclusion, guère neuve, hélas, mais qu'il faut néanmoins répéter, tant le déséquilibre persiste: les femmes sont quasi absentes de la sphère publique parce qu'elles sont encore responsables en très grande partie de la sphère privée. Naguère, ce partage passait pour naturel, les hautes vertus morales des femmes les destinant d'emblée au foyer et les écartant du bruit et de la fureur de la vie politique ${ }^{2}$. De nos jours, cette vision des choses n'a guère plus cours, et les femmes ont massivement investi le monde du travail salarié. Et pourtant, tout se passe, dans les faits, comme si nous y croyions encore. L'identification femme-foyer-enfants est des plus tenaces.

Elles sont en retard d'une révolution, dira-t-on, celles qui affirment qu'entre les femmes et le pouvoir, se dressent des barrières souvent insurmontables. Voyez Kim Campbell, voyez Lise Bissonnette. Les femmes sont maintenant partout, et leur nombre ne cessera d'augmenter.

Pas si. sûr, répondent nos auteures, chacune à sa manière. Francine Burnonville remonte le cours de l'Histoire pour en révéler les pièges. À chaque moment d'intense mobilisation féminine visant à l'obtention de droits politiques - la Révolution française, le mouvement ouvrier du début du siècle, les années 1970 - succède une mise 
au pas des récalcitrantes et un repli, voire un recul. Impossible d'affirmer, dit-elle, que les acquis seront, cette fois, définitifs. En 1878 déjà, lors du $1^{\text {er }}$ Congrès international du droit des femmes, tenu à Paris, on réclamait le droit au travail, l'accès à tous les emplois pour les femmes, et un salaire égal pour un travail égal...

L'ouvrage Femmes et pouvoir s'attache à une seule question: quelle est la place réelle des femmes dans la sphère publique? La comparaison de deux années-repères, 1986 et 1991, permet de relever les grandes tendances. Or, n'en déplaise aux personnes pour qui tout est réglé (et pour qui le féminisme n'a plus sa raison d'être), les femmes demeurent cruellement sous-représentées dans tous les lieux de pouvoir: seulement $18,4 \%$ des députés au provincial et $17,3 \%$ au fédéral, 7,4\% des maires, 9,1\% des juges, 13,2\% des directeurs de la fonction publique, $8,8 \%$ des administrateurs des grandes institutions financières, sont des femmes. Quelles que soient les échelles, elles se trouvent au bas: elles forment $98,3 \%$ du personnel enseignant au préscolaire, $84,6 \%$ au primaire, $18,1 \%$ à l'université. Si les augmentations ont été, depuis 1986, fulgurantes dans certains cas, le nombre réel de femmes en place demeure modeste. Les aléas de la statistique font que moins il y avait de femmes au départ, plus une petite augmentation paraît spectaculaire. Un exemple: à l'École de technologie supérieure, on compte une femme sur trente-six professeurs; on pourrait donc doubler l'effectif féminin en engageant une seule personne... Il ne faut pas oublier non plus la précarité de ces progrès, que des élections, un changement de cap dans la direction, pourraient balayer. Femmes et pouvoir n'en parle pas moins d'une "Révolution tranquille" des femmes qui accèdent peu à peu aux lieux décisionnels de la sphère publique.

Le titre de l'étude de Francine Burnonville (qui aurait dû s'appeler, en toute justice, Pourquoi les femmes ne sont-elles pas allées plus loin?), semble annoncer un essai antiféministe ou, du moins, mitigé. La composition du livre est du reste curieuse: les questions passionnantes soulevées en quatrième de couverture (pourquoi les femmes n'ont-elles pas davantage investi le champ politique, pourquoi se contentent-elles encore d'emploịs subalternes, y a-t-il, chez les hommes, des stratégies défensives pour les "remettre à leur place "?) ne trouvent réponse que dans le dernier quart du livre, et encore pas entièrement, après une longue mise en place théorique et méthodologique (s'agit-il d'une thèse remaniée?) et d'un détour plus long encore par l'histoire des femmes. C'est dire que le livre annoncé et le livre réellement présenté ne coïncident pas tout à fait. Dommage, car la question de la contrainte et du choix à laquelle touche Francine 
Burnonville est des plus cruciales: dans quelle mesure les femmes choisissent-elles de vivre autrement que les hommes (travail à temps partiel, retrait du marché du travail pour s'occuper des enfants, etc.), dans quelle mesure y sont-elles forcées? Pour l'auteure, sociologue, tout est question d'habitus au sens de Bourdieu (principes permettant à une personne de se reconnaître comme faisant partie d'une classe ou d'un groupe). Les habitus de $\operatorname{sexe}^{3}$ font que les femmes mobilisent leurs énergies pour la maternité et l'amour au moment où les hommes s'occupent à constituer leurs capitaux économique et social. Leur manquent les habitus nécessaires pour être reconnues comme pouvant s'intégrer au monde des affaires ou de la politique; en outre, elles suivent des voies féminines en matière de formation qui les tiennent à l'écart, etc. Même les efforts des militantes féministes se voient souvent canalisés vers un rôle traditionnellement féminin: la prise en charge des personnes désemparées (femmes battues ou victimes d'inceste, etc.). Cause épuisante, qui ne laisse que peu de place à la revendication politique et sociale.

Y a-t-il lieu d'être optimiste? Femmes et pouvoir ouvre timidement la porte à l'espoir en invoquant la théorie de la "masse critique", selon laquelle, au-delà d'un certain seuil, les femmes au pouvoir sont suffisamment nombreuses pour modifier la culture politique et donc ouvrir la porte toute grande à leurs consœurs; par ailleurs, le principe du "plafond de verre" (une barrière invisible qui fait que la proportion de femmes plafonne à partir d'un certain niveau) continue de jouer à plein. Francine Burnonville est plus pessimiste: "l'analyse des conditions socioéconomiques des Québécoises et des Françaises ne laisse pas entrevoir un avenir radieux pour l'an 2000" (p. 233). Elle cite de nombreuses statistiques qui témoignent d'un essoufflement ou d'un recul (par exemple, les mères seules sont de plus en plus touchées par la pauvreté: $56 \%$ d'entre elles étaient pauvres en 1987, contre $46 \%$ en 1977).

Il est évident que le monde de l'emploi ne basculera pas du jour au lendemain; impossible de congédier les hommes en place pour les remplacer par des femmes ${ }^{4}$. Mais le pouvoir masculin se fissure quelque peu. L'ennui, c'est qu'il faut compter sur la volonté des pouvoirs en place - partis politiques, employeurs, syndicats - pour ouvrir la voie aux femmes. Y trouvent-ils un quelconque intérêt? En période de récession et de pénurie d'emplois, peut-on compter sur les hommes pour renoncer d'eux-mêmes à leurs privilèges? Rien n'est moins sûr. Francine Burnonville en appelle à un remaniement en profondeur des rapports entre les sexes:

Pour que disparaisse la frontière entre le monde des hommes et le monde des femmes, il faudra une nouvelle révolution dans les rapports 
privés tant au point de vue de l'expression affective et de la sexualité qu'au point de vue plus prosaïque de la prise en charge, à parts égales, des enfants et de l'aménagement de la vie quotidienne (p. 251).

Femmes et pouvoir nous offre, en conclusion, quelques propositions globales qui risquent fort de ne demeurer que des vœux pieux: des changements "doivent être apportés à la culture du pouvoir ", "une prise de conscience doit être faite sur l'iniquité qu'incarne la sousreprésentation chronique des femmes "; " des actions concrètes doivent être envisagées. (p. 47). L'absence d'agent dans toutes ces propositions révèle à quel point le mal est vaste et sournois, facile à cerner mais presque impossible à guérir. Décidément, la Révolution des femmes est très, très tranquille.

1. Francine Burnonville, Les femmes sont-elles allées trop loin? De la citoyenneté au pouvoir politique, Montréal, Le Jour, 1992, 269 p.; Lucie Desrochers, Femmes et pouvoir. La Révolution tranquille, Québec, Les Publications du Québec, 1993, 98 p.

2. Cet argument servira tout au long du $x_{x} x^{e}$ siècle, en Europe comme en Amérique du Nord, à refuser le suffrage féminin, entre autres; Henri Bourassa, LouisAdolphe Paquet, Albert Tessier le reprendront ici pendant toute la première moitié du siècle.

3. Bourdieu n'utilise pas ce terme; comme beaucoup de sociologues hommes, rappelle Francine Burdonville, il tient peu compte de la variable sexuelle dans ses analyses.

4. Mais les nouveaux organismes n'accueillent pas davantage de femmes. Les Conseils régionaux de développement, de création toute récente, ne comptent que $11,6 \%$ de femmes dans les instances décisionnelles. 